\title{
Urinary RNA test to predict kidney graft rejection
}

A molecular signature of CD $3 \varepsilon$ mRNA, interferon-inducible protein 10 (IP-10) mRNA, and $18 \mathrm{~S}$ ribosomal RNA (rRNA) levels in urinary cells can be used as a noninvasive diagnostic and prognostic test for acute cellular rejection in patients who have received kidney allografts.

In a prospective study published in the New England Journal of Medicine, Suthanthiran et al. examined 4,300 urine samples from 485 recipients of kidney grafts (taken from 3 days to 12 months after transplantation) and compared mRNA levels for a panel of genes (encoding $\mathrm{CD} 3 \varepsilon$, perforin, granzyme $\mathrm{B}$, proteinase inhibitor 9, CD103, IP-10, CXCR3, and transforming growth factor $\beta 1$ ), as well as $18 \mathrm{~S}$ rRNA levels, with allograft-rejection status.

The CD3 $\varepsilon$ mRNA:IP-10 mRNA:18S rRNA signature distinguished acute cellular rejection from acute antibodymediated rejection and borderline rejection (levels of all three markers were significantly higher in patients with biopsy specimens showing acute cellular rejection), regardless of whether patients had received anti-interleukin-2 receptor antibodies or T-cell-depleting antibodies. In addition, the signature was not affected by UTI, blood infection, or cytomegalovirus (CMV) infection, although some association with $\mathrm{BK}$ polyomavirus (BKV) infection was established.

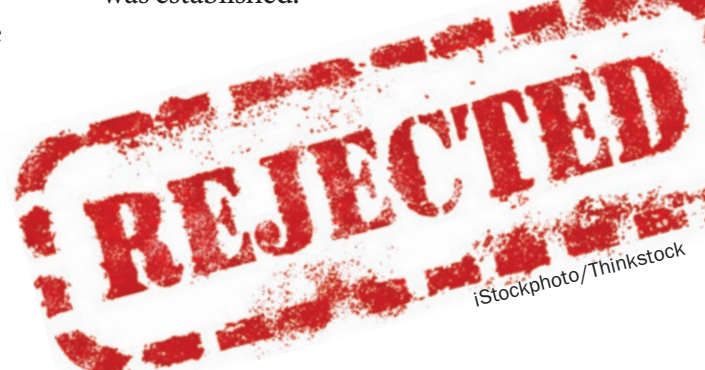

In the group of patients who did not develop acute cellular rejection, the trajectory of the signature in repeated urine samples remained relatively flat and below the diagnostic threshold, whereas in the group with rejection, a sharp rise in signature RNA levels was evident weeks before the biopsy showed rejection, suggesting that the signature could be used to monitor immune status and that immunosuppressive therapy could then be adjusted accordingly. For example, a rise in signature RNA levels prior to biopsy could serve as an indication for preemptive antirejection therapy. "Our findings suggest that the signature is a robust diagnostic biomarker for acute cellular rejection," remarks Manikkam Suthanthiran, lead investigator of the study. "Moreover, it might serve as a yardstick for the immunocompetence of the kidney-graft recipient, and facilitate personalized immunosuppressive therapy."

Melanie Clyne

Original article Suthanthiran, M. et al. Urinary-cell mRNA profile and acute cellular rejection in kidney allografts. N. Engl. J. Med. doi:10.1056/NEJMoa1215555 